Saudi Journal of Oral and Dental Research

Abbreviated Key Title: Saudi J Oral Dent Res

ISSN 2518-1300 (Print) |ISSN 2518-1297 (Online)

Scholars Middle East Publishers, Dubai, United Arab Emirates

Journal homepage: http://scholarsmepub.com/sjodr/

Review Article

\title{
Temporomandibular Joint Disorders, Management and Recent Advancements
}

\author{
Dr. Bibhu Prasad Mishra ${ }^{1}$, Dr. Stuti Gupta ${ }^{2 *}$, Dr. Deepak Tanwar
}

${ }^{1}$ Post Graduate IIIrd year, ITS Dental College, Hospital and Research Center, Greater Noida, Uttar Pradesh, 201308, India

${ }^{2}$ Lecturer, Department Of Periodontology and Oral Implantology, School of Dental Sciences, Sharda University, Greater Noida, Uttar Pradesh, 201308, India

${ }^{3}$ MDS,OMFS, Private Practitioner \& Consultant

DOI: $10.36348 /$ sjodr.2020.v05i01.008 $\quad$ | Received: 08.01.2020| Accepted: 15.01.2020 | Published: 19.01 .2020

*Corresponding author: Dr. Stuti Gupta

\section{Abstract}

Temporomandibular joint reconstruction (TMJR) is often required for patients with severe and/or refractory TMJ disease who have failed conservative treatment. TMJR helps improve masticatory function and is linked to improved quality of life outcomes. Alloplastic reconstruction is currently considered the treatment of choice for most serious TMJ disorders due to its many advantages, including but not limited to early recovery, consistent long-term outcomes and significant improvements in the function of the jaw. Two types of TMJR prostheses are available for reconstruction, broadly speaking: 1) inventory, and 2) custom-made prostheses. The purpose of this article is to provide a brief overview of TMJR's fundamental principles and concepts to the reader while referring to applicable existing literature.

Keyword: Alloplastic, TMJR, TMJ disorders.

Copyright @ 2020: This is an open-access article distributed under the terms of the Creative Commons Attribution license which permits unrestricted use, distribution, and reproduction in any medium for non-commercial use (NonCommercial, or CC-BY-NC) provided the original author and source are credited.

\section{INTRODUCTION}

The temporomandibular joint (TMJ) is composed of the temporal bone and the mandible, as well as a specialized dense fibrous structure, the articular disk, several ligaments, and numerous associated muscles. The TMJ is a compound joint that can be classified by anatomic type as well as by function. Anatomically the TMJ is a diarthrodial joint, which is a discontinuous articulation of two bones permitting freedom of movement that is dictated by associated muscles and limited by ligaments. Its fibrous connective tissue capsule is well innervated and well vascularized and tightly attached to the bones at the edges of their articulating surfaces. It is also a synovial joint, lined on its inner aspect by a synovial membrane, which secretes synovial fluid. The fluid acts as a joint lubricant and supplies the metabolic and nutritional needs of the nonvascularized internal joint structures. Functionally the TMJ is a compound joint, composed of four articulating surfaces: the articular facets of the temporal bone and of the mandibular condyle and the superior and inferior surfaces of the articular disk. The articular disk divides the joint into two compartments. The lower compartment permits hinge motion or rotation and hence is termed ginglymoid. The superior compartment permits sliding (or translatory) movements and is therefore called arthrodial. Hence the temporomandibular joint as a whole can be termed ginglymoarthrodial[1].

\section{CLASSIFICATION}

There is no universal classification that allows the collection of standard data that can be used to compare the various techniques published in the literature.

Reasons for classification-Universal medical code. It is from these codes that data can be collected and analysed for the purpose of providing the hard evidence needed to determine whether the TMJ surgery is effective in providing material benefit to patients. In other words, the hard evidence for TMJ surgery can only be secured with a universally recognized surgical classification of TMJ disorders.

Presently, there are 3 main classifications related to TMD; the Research Diagnostic Criteria (RDC) for TMD, 2 the Wilkes Classification for TMJ internal derangement, and the most recent American Academy of Orofacial Pain (AAOFP) Classification of TMD.

\section{Category 1: TMJ normal joint}

No surgery is required. In this category, a patient may present with pain specifically centred around the TMJ but report no history of locking, 
dislocation or difficulty chewing. There are no audible or palpable joint noises and the patient exhibits a full range of jaw movement with symmetrical opening. Plain films, magnetic resonance imaging (MRI) and computed tomography (CT) scans show normal joint with no radiological abnormalities. The patient may have sustained recent acute trauma following whiplash, fall or assault or experienced an ear infection. In long standing cases the TMJ arthralgia may be secondary to myofascial pain, fibromyalgia or part of a neuralgia or psychosomatic disorder. TMJ surgery has no role in these situations and patients must be carefully assessed for other ailments that may be contributing to or exacerbating the TMJ arthralgia.

\section{Category 2: TMJ minor changes}

All joint components are salvageable. In this category a patient may present with intermittent TMJ pain, joint clicking and occasional locking. Plain films demonstrate normal condyles but MRI may show mild disc displacement with reduction or excess fluid in the joint indicative of inflammation. TMJ arthrocentesis may be appropriate for cases of acute onset closed lock and TMJ arthroscopy may demonstrate mild inflammation with occasional adhesions. Both procedures may help unlock a stuck joint, but the primary treatment modality remains conservative (i.e. anti-inflammatory medication, jaw rest, soft diet).

\section{Category 3: TMJ moderate changes}

Most joint components are salvageable. In this category, patients report painful long-standing closed lock ( $>2$ months), joint swelling or painful recur-rent dislocation of the TMJ. The patient may report difficulty chewing with moderate to severe pain levels exacerbated by jaw function. Mandibular opening is restricted either because of fear of dislocation or actual joint pain which often results in deviation of the mandible to the affected side. Because of the restricted mouth opening joint noises are often absent. While plain films may show nor-mal condylar morphology, MRI shows non-reducing disc displacement. The disc may exhibit mild contour deformity and there may be a prominent articular eminence that obstructs the backward path of the translated condyle diagnostically, the patient may have suffered an acute event such as a fracture disloca-tion of the condylar head or simply dislocation of the condyle. In long standing cases, the patient may be suffering from moderate TMJ internal derangement or synovial chondromatosis. In category 3 cases, the patient would benefit from operative TMJ arthroscopy, modified condylotomy, TMJ arthroplasty consisting of disc repositioning with or without eminectomy, or open reduction and internal fixation of displaced condylar fractures.

\section{Category 4: TMJ severe changes}

Few joint components are salvageable. In this category, patients report constant joint pain with painful crepitus and mildly limited mouth opening. Chew-ing is very painful and yawning is almost impossible without provoking severe pain. Plain films show radiological signs of early changes in condylar morphology such as flattening and beak type deformities of the condylar head which are better seen on cone-beam CT scans. MRIs demonstrate severely degenerate, dis-placed and deformed articular discs which may sometimes demonstrate perforations. Early condylar changes such as osteophytes and small subcondral cysts with loss or thinning of cartilage layer may be seen on cone-beam CT scans. The clinical picture is that of severe TMJ internal derangement with early osteoarthritis. This category may also include rare metabolic, inflammatory or developmental disorders of the TMJ. TMJ discectomy with or without debridement, shaving or surgical reduction of the condylar head, articular eminence and glenoid fossa is the mainstay of treatment.

\section{Category 5: TMJ catastrophic changes}

No joint components are salvageable. Patients in this category report intolerable low grade joint pain and joint crepitus with constant locking and inability to chew anything solid. Plain films show obvious degenerative changes to the condyle which is better depicted on cone-beam CT scans which show irregular articular surface and large subchondral cysts. MRI shows severely degenerated disc which is often difficult to visualize with low signal from the condyle that appears irregular and deformed. Diagnos-tically, these patients suffer from TMJ osteoarthritis or degenerative joint disease that may be the result of multiple previous operations. Where the joint pain is absent or tolerable, the patient may have TMJ osteoarthrosis or in rare cases, TMJ ankylosis, condylysis or tumour. Patients in this category would benefit from condylectomy, discectomy and total joint replacement where feasible.

\section{Imaging modalities for temporomandibular joint disorders}

The diagnosis and management of temporomandibular disorders (TMD) require both clinical and imaging examinations of the temporomandibular joint (TMJ). A variety of modalities can be used to image the TMJ, including magnetic resonance imaging (MRI), computed tomography (CT), cone beam CT, ultrasonography, conventional radiography. The outlines that indicate the most frequently used imaging techniques in TMD diagnosis. Cone beam CT provides high resolution multiplanar reconstruction of the TMJ, with a low radiation dose, without superimposition of the bony structures. MRI is a noninvasive technique, considered to be the gold standard in imaging the soft tissue components of the TMJ. MRI is used to evaluate the articular disc in terms of location and morphology. The early signs of TMD and the presence of joint effusion can be determined.

Panoramic
radiography-Panoramic
radiography can help evaluate the following:


degenerative bone changes (only in late stages; it is inadequate for the early detection of osseous modifications); asymmetries of the condyles, hyperplasia, hypoplasia, trauma, tumors.

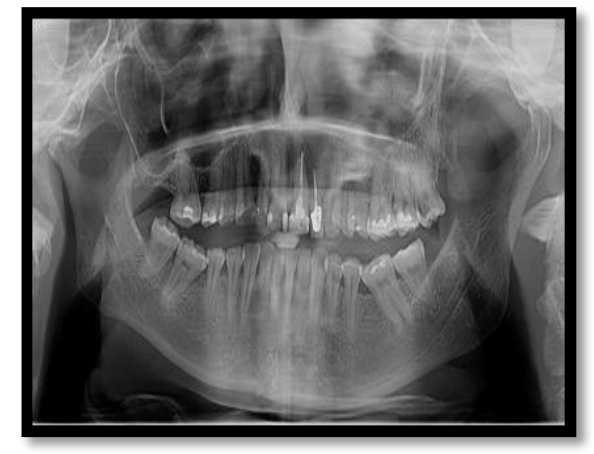

Fig-1: Panoramic radiography: important asymmetry between right and left mandibular condyle

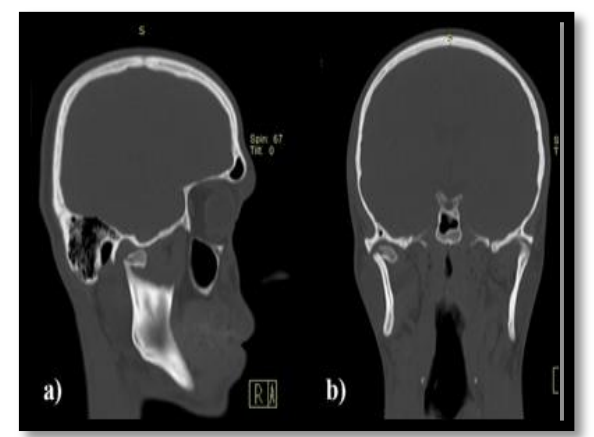

Fig-2: CT scan of an intracapsular fracture of the right TMJ

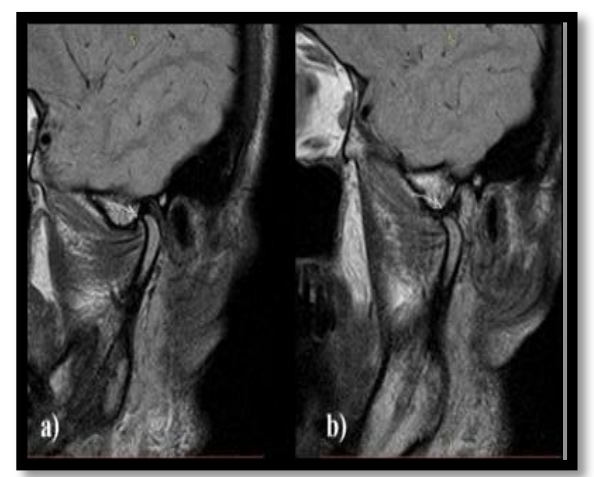

Fig-3: Sagittal, proton density, MRI of a normal TMJ

\section{DISORDERS}

Taxonomic

classification

for temporomandibular disorder- Temporomandibular joint articular disorder, Masticatory muscle disorders, Headache disorders, associated structure

Taxonomic classification for temporomandibular joint articular disorders- Joint pain, Arthralgia, Arthritis disorders
- Disk displacement with reduction, ii. Disk displacement with reduction with intermittent locking,

- Disk displacement without reduction with limited mouth opening, IV. Disk displacement without reduction

Joint diseases- Degenerative joint disease (osteoarthrosis, osteoarthritis), Condylysis, Osteochondritis dissecans, Osteonecrosis, Systemic arthritides, Neoplasms, Synovial chondromatosis

Fractures- Congenital/developmental disorders, Aplasia, Hypoplasia, Hyperplas

Taxonomic classification for masticatory muscle disorders- Muscle pain limited to the orofacial region, Myalgia, Local myalgia, Myofascial pain with spreading, and Myofascial pain with referral [1].

\section{Conservative treatment}

The main goals of treatment for temporomandibular disorders (TMD) are to reduce or eliminate pain and/or joint noises, and to restore normal mandibular function. It is well established in the literature that the majority of patients with TMD achieve good relief of symptoms with conservative measures such as occlusal splint.

\section{Pharmacotherapy}

Medications are often prescribed in conjunction with other forms of treatment for TMD. If used properly, pharmacotherapy can be a valuable adjunctive aid to the relief of symptoms when prescribed as part of a comprehensive management programme.

\section{Non-steroidal anti-inflammatory drugs (NSAID)}

The analgesic effect is specific only in cases of TMD where pain is the result of an inflammatory process such as synovitis or myositis. Aspirin, ibuprofen and paracetamol are some of the more commonly used drugs in this class, although paracetamol has poor anti-inflammatory properties, and is only used in combination with opioids for TMD. Side effects include gastric irritation, allergies, and liver dysfunction.

\section{Narcotics}

Opiate narcotics act on specific opioid receptor sites in the central nervous system conferring a central analgesic effect that, unlike NSAIDs, is not confined to pain arising from inflammatory processes. At usual clinical doses, narcotics are more effective in dampening the patient's emotional response to pain rather than eliminating the pain sensation itself; that is, the patient will still report the presence of pain, but will be better able to tolerate it after narcotic administration. Codeine, oxycodone, hydrocodone and propoxyphene 
are some of the more commonly prescribed narcotics that can be taken orally.

\section{Muscle relaxants}

Centrally-acting muscle relaxants are occasionally used for the treatment of spasmodic muscle pain associated with myofascial pain dysfunction (MPD). It is often difficult to ascertain, however, whether the major analgesic effect comes from their selective effect on relieving muscle spasm or from their general action as a sedative.

\section{Tranquilizers}

In the presence of high levels of emotional stress associated with TMD, tranquilizing agents such as benzodiazepines, or less commonly phenothiazines, are used to help the patient cope with stress by helping reduce the perception or reaction to stress. Tranquilizers such as diazepam are commonly used as supportive therapy, but because of the high potential for dependency, should not be prescribed for more than a consecutive ten-day period. The duration may be increased up to three weeks if it is administered only at bedtime as a muscle relaxant to help control nocturnal bruxism.

\section{Antidepressants}

In low doses, tricyclic antidepressants such as amytriptyline, doxepin and imipramine, have been shown to be beneficial in the treatment of chronic orofacial pain as it is often found in long-standing TMD cases. The analgesic properties are independent of the anti-depressant effect, which requires higher doses. Therapeutically, these drugs are particularly useful for TMD patients with chronic pain, sleep disturbance and depression.

\section{Occlusal therapy}

Although dental occlusion has never been proven to be an aetiological factor in TMD, it has nonetheless been referred to as a contributing factor in the course of the disease. Malocclusions, occlusal interferences, centric relation-centric occlusion discrepancies, lack of dental support (missing teeth) have all been implicated as contributing or exacerbating factors in patients who are predisposed to TMD.

\section{Occlusal appliance therapy}

The most common form of treatment provided by dentists for TMD is occlusal appliance therapy (OAT). This may be otherwise referred to as a biteraising appliance, occlusal splint, bite guard, or even interocclusal orthopaedic device. An occlusal appliance is a removable device, usually made of hard acrylic that is custom-made to fit over the occlusal surfaces of the teeth in one arch, either the mandible or maxilla.

There are generally two types of occlusal appliances in common use: the flat plane (stabilization) appliance, and the anterior repositioning appliance. The flat plane appliance covers all the teeth in the arch and its purpose is to relax the elevator muscles, provide joint stability and to protect the teeth from abnormal forces such as bruxism. There must be bilateral, equal intensity posterior occlusal contacts so that an environment of a stable physiological mandibular posture is created. Canine guidance should be created for lateral and protrusive excursions of the mandible.

\section{Occlusal adjustment}

There is a limited role for occlusal adjustment or selective tooth grinding in the treatment of TMD which, in effect, is an irreversible treatment modality." It is probably best confined to situations where acute TMD symptoms arise from rapid changes in occlusion following recent restorative dental treatment, such as a 'high spot' on an amalgam restoration, changes in condylar integrity secondary to disease (for example, condylysis), or postorthognathic surgery, so as to redistribute the adverse occlusal loading forces as they occur.

\section{Physiotherapy}

The aim of physiotherapy is to restore normal mandibular function by a number of physical techniques that serve to relieve musculoskeletal pain and promote healing of tissues

\section{Joint mobilization}

Physical manipulation by passive joint stretching and joint distraction can be used in cases of restricted mouth opening resulting from MPD and/or internal derangement.

The aim is to passively restore joint motion and improve joint function by repeated digital manipulation of the jaws by the physiotherapist. Prior to manipulation, it is essential that the patient's symptoms of pain and muscle spasm are brought under control by other means.

\section{Jaw exercises}

Jaw exercises are helpful in increasing muscle strength and co-ordination, and increasing the stability and range of motion of the TMJs. There are three basic types of exercise, each of which serves a particular purpose. Musclestrength is addressed by isometric exercises whilst isotonic exercises are used to increase range of motion. Coordination of muscle function is achieved by repetitive rhythmic exercises.

\section{Physical modalities}

The most common physical modalities used by physiotherapists for the treatment of TMD are ultrasound, short-wave diathermy, superficial heat and cold, transcutaneous electrical nerve stimulation (TENSY and, more recently, ionophoresis. The use of heat, particularly applied to areas of muscle spasm, can help relax the muscle and increase blood flow within it. 
The simplest measure is moist heat applied via a heat compress over the painful areas.

Short-wave diathermy and ultrasound are simply an extension of this technique of heat therapy. Short-wave diathermy only provides heat to superficial tissues whereas ultrasound can transmit heat through tissues to a depth of $50 \mathrm{~mm}$. The purpose is to alleviate chronic pain and increase tissue extensibility.

Transcutaneous electrical nerve stimulation uses a low-voltage electrical current that is designed for sensory counter-stimulation in painful disorders. It is used to decrease muscle pain and hyperactivity and may also be useful in neuromuscular re-education. Ionophoresis utilizes a low-voltage direct current generator to push like-charged ions of therapeutic substances such as dexamethasone into tender, swollen tissues.

PHYSICAL THERAPY- Physical therapy is a noninvasive, conservative therapy that helps to relieve musculoskeletal pain and restore function by altering sensory input, reducing inflammation, increasing mandibular range of motion, promoting repair and regeneration of tissue, and helping in reestablishment of oral motor function.

Types of physical therapy include manual manipulation, massage, TMJ distraction and mobilization, therapeutic exercises, coolant therapy (spray and stretch technique), photobiomodulation, ultrasound therapy, ionophoresis, and transcutaneous electrical nerve stimulation (TENS). Ionophoresis is a technique that is used to carry drug ions across a tissue barrier. A weak current is used to enhance transport of the medication through the skin to deeper tissues. It has been proposed to be beneficial in management of inflammatory TMJ disease disorders such as juvenile idiopathic arthritis. However, literature is limited by a lack of randomized controlled trials, control groups, and short-term follow-up.

TENS therapy uses a low-voltage, lowamperage biphasic current of varying frequency. As an adjunctive therapy, it has been shown to be effective in improving TMJ function by reducing pain and improving range of motion in patients with TMJ arthralgia and disc displacement disorders. However, similar to other physical therapy modalities, literature is limited by the presence of multiple methodological limitations. Photobiomodulation therapy or low-level laser therapy (LLLT) is a therapeutic modality that generates light of a single wavelength. Exposure to LLLT results in photochemical reactions within the cells, which is referred to as photobiomodulation or photobiostimulation. LLLT involves the use of visible red or near-infrared light. Light energy is absorbed within the cells by cellular photoreceptors called cytochromophores. There are various types of LLLT devices available. They are primarily characterized based on the element used for generating the light. Photobiomodulation therapy has been suggested to be effective in management of TMJ arthralgia, inflammatory joint diseases, and/or degenerative joint disease.

\section{Pharmacological management MEDICINE INDICATION DOSAGE-} Ibuprofen TMJ pain, TMJ degenerative joint disease Infants and children (6 mo to 12 y old): 5-10 $\mathrm{mg} / \mathrm{kg}$ orally every $6-8 \mathrm{~h}$ as needed. Maximum of 4 doses/d Adolescents (_12 y) 200-400 mg orally every 4-6 h as needed. Maximum of $1200 \mathrm{mg} / \mathrm{d}$ Naproxen TMJ pain, TMJ degenerative joint disease Infants $(<2$ y old $)$ : safety and efficacy of naproxen has not been established Children $(25 \mathrm{~kg}$ or greater) $10 \mathrm{mg} / \mathrm{kg} / \mathrm{d}$ orally in 2 divided doses.

Adolescents (12 y of age): $220 \mathrm{mg}$ every 8-12 $\mathrm{h}$ as needed with maximum dose of $660 \mathrm{mg}$ in $24 \mathrm{~h}$ Duration of therapy should not be longer than $10 \mathrm{~d}$ Diclofenan TMJ pain, TMJ degenerative joint disease Infants: safety and efficacy have not been established Children and adolescents <18 y old: $2-3 \mathrm{mg} / \mathrm{kg} / \mathrm{d}$ in $2-4$ divided doses. Maximum of $200 \mathrm{mg} / \mathrm{d}$.

Available as topical preparation- Meloxicam TMJ pain, TMJ degenerative joint disease Infants: safety and efficacy not been established Children and adolescents: $0.125 \mathrm{mg} / \mathrm{kg}$ orally once daily with maximum of $7.5 \mathrm{mg}$ Celecoxib TMJ pain, TMJ degenerative joint disease. Infants: safety and efficacy have not been established Children ( $2 \mathrm{y}$ and older, 10-25 kg): $50 \mathrm{mg}$ orally twice daily Children and adolescents (25 kg or more): $100 \mathrm{mg}$ orally twicedaily Acetaminophen TMJ pain, TMJ degenerative joint disease Infants and children $(<60 \quad \mathrm{~kg}) \quad 10-15$ $\mathrm{mg} / \mathrm{kg} /$ dose orally every $4-6 \mathrm{~h}$, with maximum of 1625 $\mathrm{mg} / \mathrm{d}$,Adolescents (60 kg or greater) $650 \mathrm{mg}$ orally every 4-6 h, as needed. Maximum dose of 3250 $\mathrm{mg} / \mathrm{d}[31]$.

\section{ORTHOTIC JAW APPLIANCE THERAPY}

Orthotic jaw appliance therapy refers to customized acrylic devices that fit onto maxillary teeth, mandibular teeth, or both. They are available in a variety of materials and designs. The most common types are (1) stabilization appliances of hard, soft, or dual acrylic; (2) anterior positioning appliances; and (3) anterior bite appliances.

The purpose of orthotic jaw appliance therapy is to reduce TMJ-related pain, increase range of motion, and improve joint function. The exact mechanism of action of these devices is unknown. It is suggested that orthotic occlusal device therapy may alter the TMJ mechanics and increase joint mobility, enhance patient awareness of oral parafunctional behaviors, disrupt neuromuscular that determines TMJ-fossa 
relationships, and protect teeth and restorations from jaw clenching, causing potential fracture or attrition of dentition[37].

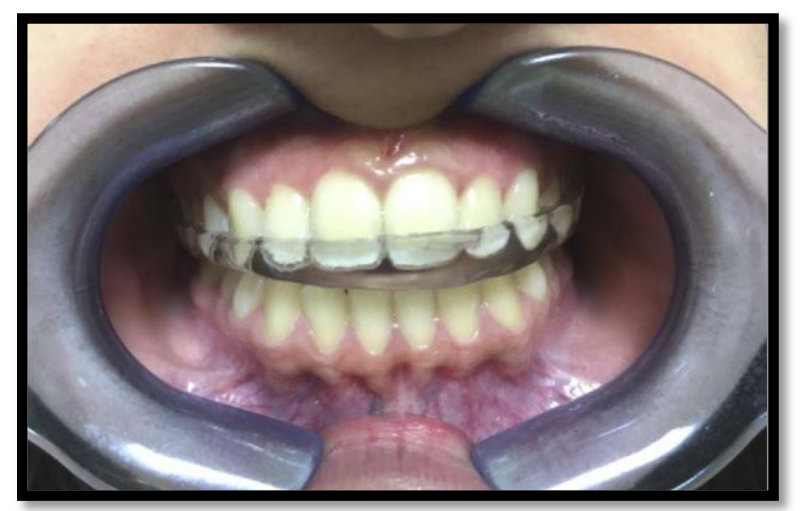

Fig-4: Full-coverage hard stabilization orthotic jaw appliance. It has a flat occlusal surface to allow uniform posterior and anterior contacts with the opposing teeth on closure [37]

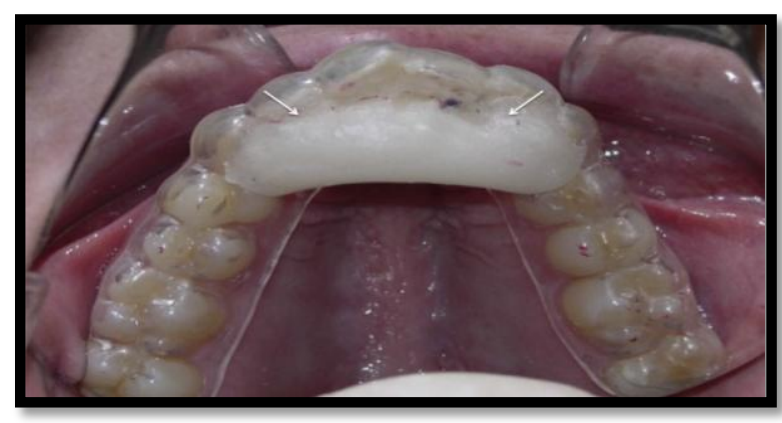

Fig-5: Anterior repositioning appliance, or MORA. Note the reverse anterior ramp (arrows) on the palatal section of the anterior segment of the device. It functions as a guide to place the mandible into a protrusive position [37]

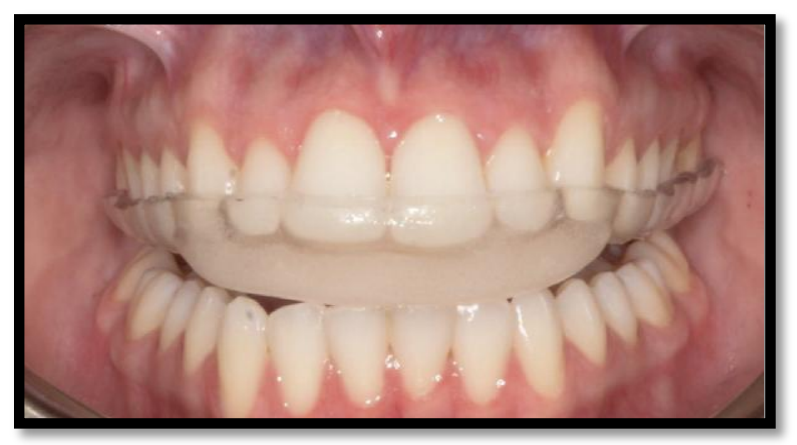

Fig-6: Full-coverage anterior bite appliance. This device allows only anterior teeth of the opposing arch to contact on closure

\section{CONCLUSION}

TMD is a complex disorder with common presenting signs and symptoms. This topic presented nonsurgical strategies used to alleviate the pain and dysfunction associated with the TMJ. Since an exact correlation between diagnosis and treatment is not always possible, success or failure with nonsurgical modalities is not a reliable outcome, even though this therapy may aid in diagnosis and be the first step for most patients. When surgery is indicated or evidence- based, nonsurgical techniques are a crucial adjunct perioperatively, if not forever. Remarkably good success has been reported with several surgical procedures, which differ in their fundamental approach to the problem and their aggressiveness. Most of these techniques share common denominators: first, a lateral approach to the capsule and ligament; second, a severing of the posterior attachment-disk attachments to the capsule once the superior joint space is accessed; and, third, a blunt delineation of the joint spaces. Although the capsule and ligament tissues are approximated at the conclusion of the procedure, the patient is encouraged to function on the operated joint. Long before arthroscopic surgery, Toller recognized the importance of mobilizing the condyle-disk-fossa relations to achieve a successful result. He devised the lateral capsular arrangement procedure. It remains to be determined whether disk repositioning, posterior attachment repair, diskectomy, high condylectomy, and even condylotomy derive some or all of their therapeutic benefits through a lateral capsule and ligament release and mobilization of the disk complex. Arthrocentesis and arthroscopic surgical procedures for treatment of the closed lock condition appear to be therapeutic through the same mechanism. Open surgical approaches to TMJ internal derangements are now relegated to a tertiary line of care following nonsurgical therapy and arthrocentesis/arthroscopy for most conditions. They do, however, have a clear indication for certain mechanical conditions directly attributed to a disk obstruction. Now, as we regress with progress, arthrocentesis with and without steroid injection, a procedure performed by many surgeons years before the pathology of the joint was even elucidated, has become a mainstay for treatment. This treatment alone has significantly reduced the need to intervene via arthrotomy. The patient with end-stage TMJ disease is typically afflicted by severe unrelenting pain, restricted jaw function, facial deformity, depression, compromised interpersonal relationships, and financial hardships. Given the complexities involved, these patients pose a significant challenge for the most experienced clinicians. A coordinated team of specialist's best provides optimum care.

\section{REFERENCES}

1. Dimitroulis, G. (2013). A new surgical classification for temporomandibular joint disorders. International journal of oral and maxillofacial surgery, 42(2), 218-222.

2. Mercuri, L. G. (2016). Temporomandibular joint total joint replacement-TMJ TJR. Switzerland: Springer Int Pub.

3. Laplanche, O., Ehrmann, E., Pedeutour, P., \& Duminil, G. (2012). TMD clinical diagnostic classification (Temporo Mandibular Disorders). Journal of Dentofacial Anomalies and Orthodontics, 15(2). 
Bibhu Prasad Mishra \& Stuti Gupta; Saudi J Oral Dent Res, Jan 2020; 5(1): 48-55

4. Devi Devaraj, S., \& Pradeep, D. Internal Derangement of Temporomandibular Joint-A Review.

5. George, D., MDSc(Melb), FDSRCS(Eng), FFDRCS(Irel), Henry, Gremillion, DDST, M. (1995). Franklin Dolwick, DMD, PhDS John H. Walter, PT, OCS, Temporomandibular disorders. 2. Non-surgical treatment, Australian Dental Journal, 40(6):372-6.

6. Posselt, U. (1968). "Physiology of occlusion and rehabilitation". Oxford, Edinburgh: Blackwell Scientific Publications.

7. Scrivani, S. J., Khawaja, S. N., \& Bavia, P. F. (2018). Nonsurgical management of pediatric temporomandibular joint dysfunction. Oral and Maxillofacial Surgery Clinics, 30(1), 35-45.

8. Sathe, N. U., Bhange, P., Acharya, R., Bhatia, A., \& Mhashal, S. (2011). Interposition arthroplasty of temporomandibular joint ankylosis using temporalis muscle flap: our experience. Surgical Techniques Development, 1(1), e19-e19.

9. Goswami, D., Singh, S., Bhutia, O., Baidya, D., \& Sawhney, C. (2016). Management of young patients with temporomandibular joint ankylosisa surgical and anesthetic challenge. Indian Journal of Surgery, 78(6), 482-489.

10. Talmaceanu, D., Lenghel, L. M., Bolog, N., Hedesiu, M., Buduru, S., Rotar, H., ... \& Baciut, G. (2018). Imaging modalities for temporomandibular joint disorders: an update. Clujul Medical, 91(3), 280.

11. Miller, D.B.(2011). Diagnosis and treatment of temporomandibular disorders: emergence of a new care guidelines statement. Oral Surg Oral Med Oral Pathol Oral Radiol Endod. Feb;111(2):133;

12. Guarda-Nardini, L., Manfredini, D., \& Ferronato, G. (2008). Total temporomandibular joint replacement: a clinical case with a proposal for post-surgical rehabilitation. Journal of CranioMaxillofacial Surgery, 36(7), 403-409.

13. Kaban, L. B., Bouchard, C., \& Troulis, M. J. (2009). A protocol for management of temporomandibular joint ankylosis in children. Journal of Oral and Maxillofacial Surgery, 67(9), 1966-1978.

14. Reza, M., DMDa, Louis, G., Mercuri, DDS, MSB. (2015). Management of Temporomandibular Joint Ankylosis, Oral Maxillofacial Surg Clin N Am, 27(2015) 27-35.

15. Rajan, R., Reddy, N. V. V., Potturi, A., Jhawar, D., Muralidhar, P. V., \& Reddy, B. (2014). Gap arthroplasty of temporomandibular joint ankylosis by transoral access: a case series. International journal of oral and maxillofacial surgery, 43(12), 1468-1472.

16. Shaffer, S. M., Brismée, J. M., Sizer, P. S., \& Courtney, C. A. (2014). Temporomandibular disorders. Part 1: anatomy and examination/diagnosis. Journal of manual \& manipulative therapy, 22(1), 2-12.
17. Tuncel, U., \& Ozgenel, G. Y. (2011). Use of human amniotic membrane as an interpositional material in treatment of temporomandibular joint ankylosis. Journal of Oral and Maxillofacial Surgery, 69(6), e58-e66.

18. Abramowicz, S., Kim, S., Prahalad, S., Chouinard, A. F., \& Kaban, L. B. (2016). Juvenile arthritis: current concepts in terminology, etiopathogenesis, diagnosis, and management. International journal of oral and maxillofacial surgery, 45(7), 801-812.

19. Durham, J., \& Wassell, R. W. (2011). Recent advancements in temporomandibular disorders (TMDs). Reviews in pain, 5(1), 18-25.

20. Ebrahimi, A., \& Ashford, B. G. (2010). Advances in temporomandibular joint reconstruction. Current opinion in otolaryngology \& head and neck surgery, 18(4), 255-260.

21. Goldstein, B. H. (1999). Temporomandibular disorders: a review of current understanding. Oral Surgery, Oral Medicine, Oral Pathology, Oral Radiology, and Endodontology, 88(4), 379-385.

22. Indresano, A. T., \& Mobati, D. A. (2006). History of temporomandibular joint surgery. Oral and Maxillofacial Surgery Clinics, 18(3), 283-289.

23. Kraus, S. (2007). Temporomandibular disorders, head and orofacial pain: cervical spine considerations. Dental Clinics, 51(1), 161-193.

24. Kreutziger, K. L. (1984). Surgery of the temporomandibular joint. I. Surgical anatomy and surgical incisions. Oral surgery, oral medicine, oral pathology, 58(6), 637-646.

25. Laviv, A., Sadow, P. M., \& Keith, D. A. (2015). Pseudogout in the temporomandibular joint with imaging, arthroscopic, operative, and pathologic findings. Report of an unusual case. Journal of Oral and Maxillofacial Surgery, 73(6), 1106-1112.

26. Manfredini, D., Segù, M., Arveda, N., Lombardo, L., Siciliani, G., Rossi, A., \& Guarda-Nardini, L. (2016). Temporomandibular joint disorders in patients with different facial morphology. A systematic review of the literature. Journal of Oral and Maxillofacial Surgery, 74(1), 29-46.

27. McNeill, C. (1983). Craniomandibular (TMJ) disorders-The state of the art. Part II: Accepted diagnostic and treatment modalities. Journal of Prosthetic Dentistry, 49(3), 393-397.

28. Moses, J. J., Sartoris, D., Glass, R., Tanaka, T., \& Poker, I. (1989). The effect of arthroscopic surgical lysis and lavage of the superior joint space on TMJ disc position and mobility. Journal of Oral and Maxillofacial Surgery, 47(7), 674-678.

29. McCain, J. P., Hossameldin, R. H., Srouji, S., \& Maher, A. (2015). Arthroscopic discopexy is effective in managing temporomandibular joint internal derangement in patients with Wilkes stage II and III. Journal of Oral and Maxillofacial Surgery, 73(3), 391-401.

30. Abboud, W., Yahalom, R., \& Givol, N. (2015). Treatment of intermittent locking of the jaw in Wilkes stage II derangement by arthroscopic lysis 
and lavage. Journal of Oral and Maxillofacial Surgery, 73(8), 1466-1472.

31. Heir, G. M. (2018). The efficacy of pharmacologic treatment of temporomandibular disorders. Oral and maxillofacial surgery clinics of North America, 30(3), 279-285.

32. Bae, S. S., \& Aronovich, S. (2018). Trauma to the Pediatric Temporomandibular Joint. Oral and maxillofacial surgery clinics of North America, 30(1), 47-60.

33. Bender, M. E., Lipin, R. B., \& Goudy, S. L. (2018). Development of the pediatric temporomandibular joint. Oral and Maxillofacial Surgery Clinics, 30(1), 1-9.

34. Bouloux, G. F. (2018). The Use of Synovial Fluid Analysis for Diagnosis of Temporomandibular Joint Disorders. Oral and maxillofacial surgery clinics of North America, 30(3), 251-256.

35. Dolwick, M. F., \& Widmer, C. G. (2018). Orthognathic Surgery as a Treatment for Temporomandibular Disorders. Oral and Maxillofacial Surgery Clinics, 30(3), 303-323.

36. Graff-Radford, S. B., \& Abbott, J. J. (2016). Temporomandibular disorders and headache. Oral and Maxillofacial Surgery Clinics, 28(3), 335-349.

37. Greene, C. S., \& Menchel, H. F. (2018). The use of oral appliances in the management of temporomandibular disorders. Oral and Maxillofacial Surgery Clinics, 30(3), 265-277.

38. Horswell, B. B., \& Sheikh, J. (2018). Evaluation of pain syndromes, headache, and temporomandibular joint disorders in children. Oral and Maxillofacial Surgery Clinics, 30(1), 11-24.

39. Kim, S., \& Keith, D. A. (2018). Combined or Staged Temporomandibular Joint and Orthognathic Surgery for Patients with Internal Derangement and Dentofacial Deformities. Oral and maxillofacial surgery clinics of North America, 30(3), 351-354.

40. Koslin, M. (2004). Laser applications in temporomandibular joint arthroscopic surgery. Oral and Maxillofacial Surgery Clinics, 16(2), 269-275.

41. Larheim, T. A., Hol, C., Ottersen, M. K., MorkKnutsen, B. B., \& Arvidsson, L. Z. (2018). The role of imaging in the diagnosis of temporomandibular joint pathology. Oral and Maxillofacial Surgery Clinics, 30(3), 239-249.
42. Laskin, D. M. (2018). Arthroscopy Versus Arthrocentesis for Treating Internal Derangements of the Temporomandibular Joint. Oral and Maxillofacial Surgery Clinics, 30(3), 325-328.

43. Manfredini, D. (2018). Occlusal Equilibration for the Management of Temporomandibular Disorders. Oral and maxillofacial surgery clinics of North America, 30(3), 257-264.

44. Mercuri, L. G. (2018). Costochondral Graft Versus Total Alloplastic Joint for Temporomandibular Joint Reconstruction. Oral and maxillofacial surgery clinics of North America, 30(3), 335-342.

45. Nadershah, M., \& Mehra, P. (2015). Orthognathic surgery in the presence of temporomandibular dysfunction: what happens next?. Oral and Maxillofacial Surgery Clinics, 27(1), 11-26.

46. Prechel, U., Ottl, P., Ahlers, O. M., \& Neff, A. (2018). The Treatment of Temporomandibular Joint Dislocation: A Systematic Review. Deutsches Aerzteblatt International, 115(5), 59.

47. Renapurkar, S. K. (2018). Discectomy versus disc preservation for internal derangement of the temporomandibular joint. Oral and Maxillofacial Surgery Clinics, 30(3), 329-333.

48. Resnick, C. M. (2018). Temporomandibular joint reconstruction in the growing child. Oral and Maxillofacial Surgery Clinics, 30(1), 109-121.

49. Scrivani, S. J., Khawaja, S. N., \& Bavia, P. F. (2018). Nonsurgical management of pediatric temporomandibular joint dysfunction. Oral and Maxillofacial Surgery Clinics, 30(1), 35-45.

50. Shroff, B. (2018). Malocclusion as a Cause for Temporomandibular Disorders and Orthodontics as a Treatment. Oral and maxillofacial surgery clinics of North America, 30(3), 299-302.

51. Movahed, R., Teschke, M., \& Wolford, L. M. (2013). Protocol for concomitant temporomandibular joint custom-fitted total joint reconstruction and orthognathic surgery utilizing computer-assisted surgical simulation. Journal of Oral and Maxillofacial Surgery,71(12), 21232129.

52. González-García, R. (2015). The current role and the future of minimally invasive temporomandibular joint surgery. Oral and Maxillofacial Surgery Clinics, 27(1), 69-84. 